\title{
Breakthrough cancer pain - still a challenge
}

This article was published in the following Dove Press journal:

Journal of Pain Research

16 November 2012

Number of times this article has been viewed

\section{Cesar Margarit ${ }^{1}$ \\ Joaquim Juliá ${ }^{2}$ \\ Rafael López ${ }^{3}$ \\ Antonio Anton ${ }^{4}$ \\ Yolanda Escobar ${ }^{5}$ \\ Ana Casas ${ }^{6}$ \\ Juan Jesús $\mathrm{Cruz}^{7}$ \\ Rafael Galvez ${ }^{8}$ \\ Ana Mañas ${ }^{9}$ \\ Francisco Zaragozá ${ }^{10}$ \\ 'Pain Unit, Alicante University General Hospital, Alicante, Spain; ${ }^{2}$ Department of Integral Support- Palliative Care, Catalan Institute of Oncology (ICO), Germans Trias i Pujol University Hospital, Badalona, Spain; ${ }^{3}$ Department of Clinical Oncology, University Hospital Complex, Santiago de Compostela, Spain; ${ }^{4}$ Department of Clinical Oncology, Miguel Servet Hospital, Zaragoza, Spain; ${ }^{5}$ Department of Clinical Oncology, Gregorio Marañón Hospital, Madrid, Spain; ${ }^{6}$ Department of Oncology, Virgen Macarena Hospital, Seville, Spain; ${ }^{7}$ Department of Clinical Oncology, Salamanca Hospital, Salamanca, Spain; ${ }^{8}$ Pain and Palliative Care Unit, Virgen de las Nieves Hospital, Granada, Spain; ' Department of Oncology-Radiotherapy, La Paz Hospital, Madrid, Spain; ${ }^{10}$ Department of Pharmacology, University of Alcalá de Henares, Spain}

Correspondence: Cesar Margarit Unidad del Dolor, Hospital General Universitario de Alicante, C/ Maestro Alonso 103, 03010 Alicante, Spain Email cmargarit69@gmail.com
Abstract: Breakthrough cancer pain is defined as transient pain exacerbation in patients with stable and controlled basal pain. Although variable, the prevalence of breakthrough cancer pain is high (33\%-95\%). According to the American Pain Foundation, breakthrough pain is observed in $50 \%-90 \%$ of all hospitalized cancer patients, in $89 \%$ of all patients admitted to homes for the elderly and terminal-patient care centers, and in $35 \%$ of all ambulatory care cancer patients. The management of breakthrough cancer pain should involve an interdisciplinary and multimodal approach. The introduction of new fentanyl formulations has represented a great advance and has notably improved treatment. Among these, the pectin-based intranasal formulation adjusts very well to the profile of breakthrough pain attacks, is effective, has a good toxicity profile, and allows for convenient dosing - affording rapid and effective analgesia with the added advantage of being easily administered by caregivers when patients are unable to collaborate.

Keywords: prevalence, management, diagnosis, opioids

\section{Introduction}

The management of neoplastic diseases undoubtedly represents one of the great medical advances of the last 20 years, in terms of both prolongation of patient survival and the availability of a growing number of increasingly individualized treatment options. This increased survival often implies a greater prevalence of symptoms of the disease (particularly pain) and a greater chronicity of such symptoms.

Despite unquestionable advances in recent years in our knowledge of the intimate physiopathological mechanisms of cancer pain, the development and upgrading of highly effective opioid drugs, and the development of increasingly effective and less invasive interventional techniques, cancer pain remains a primary challenge in the integral management of cancer patients. Initial advances resulted from the publication of the analgesic ladder in the World Health Organization (WHO) document "Cancer Pain Relief,"' which recommended the selection of analgesic drugs based on pain intensity and which was designed to allow all people to have access to optimum pain management; however the studies and publications of recent years (including those by the WHO itself) continue to emphasize that severe cancer pain remains a world public healthcare problem.

Pain is experienced by one out of every three patients with active cancer subjected to treatment and by more than three out of every four people with advanced disease. ${ }^{2}$ Persistent pain has a very important impact and greatly reduces the quality of life of cancer patients. In effect, $67 \%$ of all such patients describe the pain associated with cancer as distressing, 36\% describe it as an unbearable aspect of the disease, and 
$32 \%$ describe the pain as being so bad that they wish to die. ${ }^{3}$ Cancer patients consider health-related quality of life to be as important as life expectancy or survival, ${ }^{4}$ since cancer pain blocks interactions with family (lack of interest in the environment, feelings of isolation from the world) and with friends, and destroys personal quality of life and any perception of happiness. ${ }^{5}$

The incidence of cancer is high in Spain, approximately 400 patients per 100,000 inhabitants per year, ${ }^{5}$ with 162,000 new cases diagnosed in the year 2006 alone. ${ }^{6}$

One-half of all Spanish cancer patients survive their disease for over five years. In terms of individual risk, one out of every three Spanish males and one out of every five Spanish women will be diagnosed with cancer at some point in life. ${ }^{6}$ Considering that the prevalence of pain is very high in the advanced stages of cancer ( $70 \%-90 \%)$, it is assumed that each year, at least 75,000 people in this country will face the suffering of cancer pain, ${ }^{5}$ pain being the most feared symptom among these patients. ${ }^{5}$

Pain is generally one of the main factors contributing to insomnia in cancer patients, and in women who survive breast cancer, pain has been associated with fatigue and sleep disturbances. ${ }^{7}$ It is important to note that the ability of cancer pain to cause depression and anxiety does not appear to be related to previous depressive conditions, being an independent factor associated with pain and not the neoplastic disease. ${ }^{5}$ Severe pain is usually accompanied by fatigue, nausea, and insomnia; may adversely affect physical health; and may also produce other psychological reactions, such as anger and despair, contributing to poor mental health. ${ }^{8}$

\section{Breakthrough pain: definition, classification, and diagnosis}

The first standardized definition of breakthrough pain was established by Portenoy in $1990^{\circ}$ and is currently defined as a transient exacerbation of pain, manifesting spontaneously or related to a specific, predictable or unpredictable triggering factor, despite stable and adequately controlled basal pain. ${ }^{10-17}$ Therefore, pain episodes occurring without basal pain or with poorly controlled basal pain cannot be taken to represent breakthrough pain. ${ }^{17}$

Pain may be classified in several ways according to its etiology, severity, etc (see Table 1). In turn, breakthrough pain is classified as either spontaneous or incidental. Incidental breakthrough pain can be classified as volitional (caused by a voluntary act such as walking), nonvolitional (caused by an involuntary act such as sneezing or coughing), or procedural (caused by therapeutic interventions, for instance, the bandaging of a wound), and this classification involves therapeutic differences which are discussed in the section on treatment. ${ }^{14}$

In 2009, Davies et a ${ }^{16}$ published diagnostic criteria for breakthrough pain, such as transient pain exacerbations in the presence of adequately controlled basal pain (defined as no pain or mild pain for $>12$ hours a day during the week prior to evaluation). Given the lack of a validated clinical assessment method or tool, as used with other types of pain, the authors recommended using standardized questions to establish when pain occurs, its frequency, location, severity, duration, exacerbating factors, alleviating factors, response to analgesics, response to other interventions, the associated symptoms, and interference with the activities of daily living.

Table I Classification of chronic cancer pain

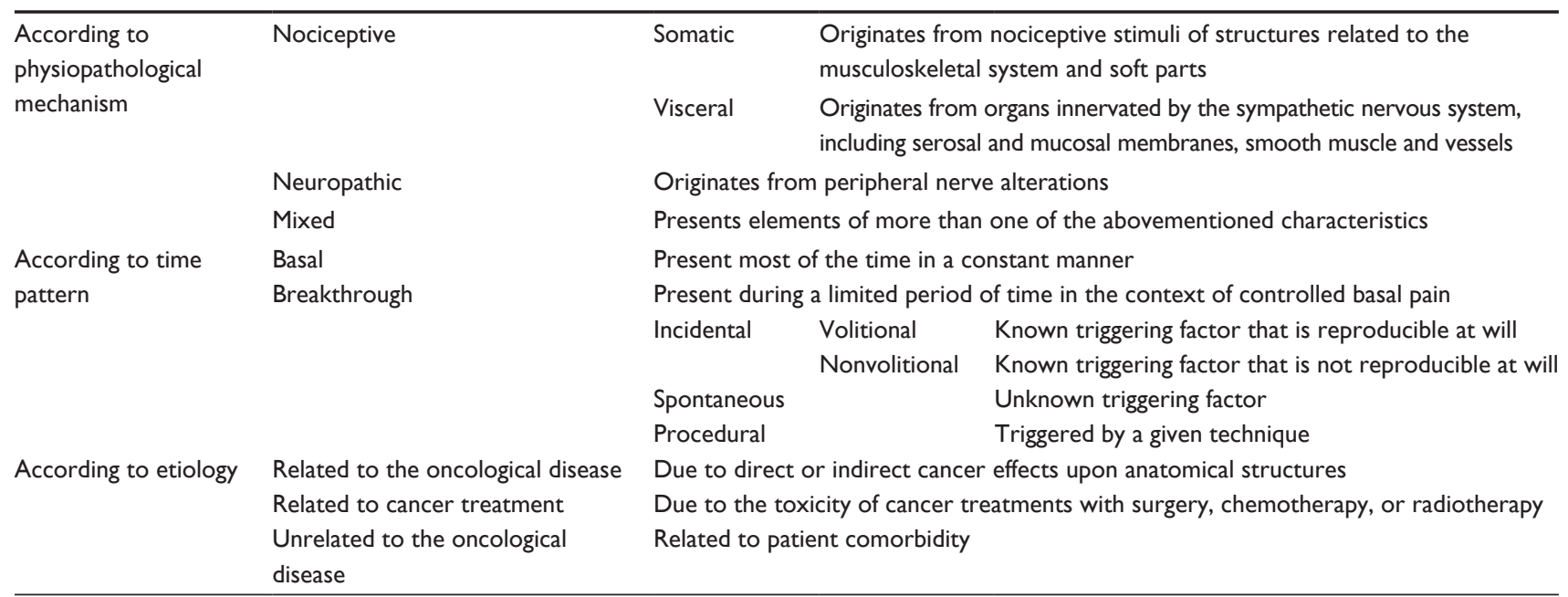


Duration of the pain is one of the differentiating characteristics of breakthrough pain, and although it can last up to 60 minutes, ${ }^{4}$ the usual duration of each episode is 15-30 minutes or even shorter. ${ }^{18,19}$ Another very important characteristic of breakthrough pain is the very brief time to maximum pain intensity: between 3-15 minutes and in some cases, only seconds. ${ }^{10,19}$ The frequency of the pain episodes varies: once a day, several times a day, or several times a week. ${ }^{20}$ The number of daily episodes is also variable, and although there is no exact number of episodes there are usually three to six; ${ }^{10,19}$ a greater number of episodes could be indicative of an uncontrolled baseline pain, forcing the revision of the basal therapeutic approach. According to different studies, the pain is unpredictable in $78.2 \%$ of cases, ${ }^{10} 53 \%$ of all breakthrough pain episodes are caused by movement and weight loading, and $7 \%$ of the episodic pain occurrences are caused by immobilization. ${ }^{18}$ Only $2 \%$ of all episodes are considered to correspond to end of dose failure, or pain that appears at the end of the analgesic effect. ${ }^{18}$ Even though the current diagnostic criteria are quite homogeneous, some differences remain that obscure comparisons of the prevalence data among the different studies. For example, Svendsen et $\mathrm{al}^{21}$ included end-of-dose failure as a form of breakthrough pain but in the latest reviews, pain due to end-of-dose failure has been removed from the classifications of breakthrough pain. These differences in the use of the breakthrough pain nomenclature may lead to error and could have complicated systematization of the clinical results. Lastly, there is a significant association between breakthrough pain and the following factors: the presence of metastases, poorer functional status, neuropathic or somatic pain, the presence of more than one type of pain, and the use of nonopioid analgesics. ${ }^{12}$ The association between the prevalence of breakthrough pain, age, and gender is more controversial; some studies have found no differences, ${ }^{12}$ but others have shown that the impact of significant breakthrough pain upon patient quality of life manifests more in younger individuals and women. ${ }^{22}$

\section{Epidemiological data and prevalence of breakthrough pain}

A considerable number of studies have been carried out in the last decade of the 20th century and the first decade of the new century with the purpose of establishing the prevalence of cancer pain. However, given the lack of unanimous diagnostic criteria and the terminological differences in the description of breakthrough pain, the conclusions drawn from these studies must be viewed with caution.
The prevalence of breakthrough cancer pain is high but quite variable because, among other reasons, the data from different studies are not fully comparable. In this context, two different studies ${ }^{9,10}$ have noted breakthrough pain on the day prior to evaluation in $63 \%$ and $51.2 \%$ respectively, of patients with controlled basal pain. The prevalence of breakthrough pain was also determined in terms of the presence of pain the day before the evaluation, in a study by Swanwick et al ${ }^{18}$ that found a prevalence of $93 \%$. However, other authors have published prevalence data on breakthrough pain without delimiting it to the day before evaluation. Thus, a $64.8 \%$ prevalence has been reported by the International Association for the Study of Pain ${ }^{12}$ versus $44 \%$ according to Davies et al ${ }^{19}$ (though the latter authors specifically refer to incidental breakthrough pain). Yet another way to report such data is represented by the pan-European survey, which found the prevalence of breakthrough pain to be very high, affecting $50 \%-90 \%$ of all hospitalized oncological patients and $63 \%$ of the patients with analgesic treatment. ${ }^{3}$

Breakthrough pain is a direct consequence of the neoplasm in $70 \%-80 \%$ of all cases and a result of anticancer treatment in $10 \%-20 \%$ of patients; in less than $10 \%$ of all cases, the pain is not related to either the malignant disease or its treatment. ${ }^{23}$ It is possible to identify triggering factors in one-half of all episodes of breakthrough pain, though not so in the remaining $50 \%{ }^{10}$

Although the prevalence data referring to cancer pain varies depending on the geographical setting, patient age, type of treatment, and other factors, the malignant disease with the greatest prevalence of pain is head and neck cancer (70\%), followed by gastrointestinal cancer (59\%), lung/ bronchial malignancies $(55 \%)$, breast cancer $(54 \%)$, and urogenital cancer $(52 \%))^{24}$

The definition and diagnostic criteria of breakthrough pain are quite recent. As a result, the present review has considered some studies on cancer pain that did not distinguish between basal pain and breakthrough pain, since some of their conclusions on the problems of cancer pain and its management are relevant. As an example, the SAGE (Systematic Assessment of Geriatric drug use via Epidemiology) study ${ }^{25}$ reported that $25 \%-40 \%$ of all elderly cancer patients in homes for the elderly experienced pain every day.

With regard to the Spanish data, a study conducted in Catalonia by Gómez-Batiste et $\mathrm{al}^{26}$ in 2002 found the prevalence of breakthrough pain to be $41 \%$, with an average frequency of one to five episodes/patient/day and an intensity of 7.3 (moderate to severe pain) on the Visual Analog Scale 
(a verbal numeric scale that ranges from $0=$ absence of pain to $10=$ worst pain imaginable). This study also found that $25 \%$ of the patients with breakthrough pain received no treatment.

As has been mentioned previously, cancer pain greatly affects patient quality of life, ${ }^{3-5}$ and this impact upon quality of life is more significant in patients with breakthrough pain. ${ }^{10}$ A study of the American Pain Foundation ${ }^{20}$ demonstrated that breakthrough pain negatively influences emotional health (in $82 \%$ of all patients), causes suffering ( $82 \%)$, wakens patients at least once a month (73\%), and influences their capacity to perform routine tasks $(76 \%)$ and their willingness to participate in activities (83\%).

In addition to the negative influence upon patient quality of life reported by the American Pain Foundation, other studies have similarly found that breakthrough pain significantly increases the hospital admission rate compared with patients without such pain $(36.9 \% \text { versus } 22.5 \% \text {, respectively })^{20,27}$ and that its presence is associated with increased levels of depression and anxiety, ${ }^{10}$ poorer physical and mental health, ${ }^{8}$ and more intense basal pain and greater functional impairment. ${ }^{12}$ An association has also been established with cancer recurrence and variations in patient survival. ${ }^{8}$ Thus, patients experiencing breakthrough pain must not only cope with the many problems and challenges posed by the diagnosis and treatment of cancer but additionally, also suffer worsened quality of life as a result of incidental pain. Despite this, a survey conducted by the American Pain Foundation ${ }^{28}$ found that $52 \%$ of all patients who complain to their physician of pain are told that breakthrough pain is a normal side effect of cancer or its treatment.

\section{Treatment of breakthrough pain}

Cancer pain treatment is not carried out on a systematic basis, and the treatment provided is moreover, suboptimum. Consequently, the results obtained are not as good as they should be. The reasons for undertreatment and for the suboptimum management of cancer pain and breakthrough pain have been examined in several studies, and in all cases, the conclusion has been that there are a number of factors involved: cultural, educational, political, religious, logistic and health resource utilization factors, ${ }^{5}$ as well as other aspects related to the patients, to the healthcare professionals, and to the healthcare system. ${ }^{20}$ Some reasons for undertreatment are shared by both patients and physicians (fear of addiction, concern about the adverse effects, and fear of tolerance), while others are inherent to patients themselves (fear of receiving bad news if reporting a worsening of pain, fear of not being regarded as a good patient). In the case of physicians, the identified barriers to adequate pain treatment are a perceived low priority of cancer pain therapy and medical-training shortcomings in this field. ${ }^{20}$ The causes attributable to the healthcare system in turn, include: the need to prioritize pain control in the healthcare system; the need for further treatment training to overcome the reluctance of healthcare professionals to prescribe opioids; the inclusion of new drug formulations within the therapeutic repertoire; and lastly, the need for increased patient awareness of the importance of adherence to therapy. It is important for healthcare professionals to contribute to such patient education, explaining how and when to take what pain medication (ie, taking medication when needed rather than waiting for the pain to become very intense; and especially, taking care to use the most appropriate medication). ${ }^{5}$

In 2011, the American Pain Foundation published two reports on breakthrough cancer pain: one describing the results of a survey involving 2000 oncologists (with a response rate of $32 \%$ ), and the other reporting data from a survey of patients with breakthrough cancer pain. In the first study, ${ }^{23}$ the oncologists pointed out an important fact: the quality of training in the management of pain during the medical career and residency is deficient ( 3 [IQR, 1 to 5] and 5 [IQR, 3 to 7], respectively; numeric rate scale of 0 to 10). According to these oncologists, the most important barriers to optimum pain control are a defective assessment of the pain, patient distrust of opioids, and physician reluctance to prescribe opioids. ${ }^{23}$ The second study ${ }^{20}$ described the opinions and problems of breakthrough pain treatment from the patient perspective. Important findings were that: $58 \%$ of the patients claimed the analgesic efficacy of breakthrough pain treatment to be inadequate; $11 \%$ of the patients with moderate to severe pain did not receive treatment for pain; $50 \%$ of patients considered that physicians did not view quality of life as an important aspect of treatment; 33\% considered that there was not enough time in the consulting office to discuss pain; and a considerable proportion (28\%) stated that the physician showed no interest in talking about this subject when asked by the patient. These statistics do not appear to differ greatly from those published almost 20 years ago by the Eastern Cooperative Oncology Group (ECOG), ${ }^{29}$ examining attitudes and medical practice in cases of cancer pain. In effect, the ECOG report concluded that the priority of pain control in cancer patients was low, and that up to one-third of all physicians preferred to wait until expected patient survival was 6 months or less, before prescribing an opioid.

A very relevant finding in the SAGE database ${ }^{25}$ was that $26 \%$ of patients with pain did not receive any analgesic 
treatment, that $16 \%$ received WHO classification first-step drugs, $32 \%$ received second-step drugs, and that only $26 \%$ received morphine. Another particularly relevant finding of this study was that the probability of receiving morphine or other potent opioids was lower in patients aged 85 years or older than in patients aged $65-74$ years (13\% versus 38\%, respectively). ${ }^{25}$

The Spanish ALGOS group ${ }^{30}$ has pointed out that the main discrepancies between ideal and actual pain management in Spain are the failure to give the patient written instructions and information, the failure to confirm whether the patient has understood the instructions, and the lack of systematic assessment of the pain. For further consideration are the results of a very recent Spanish survey ${ }^{31}$ reporting that a large percentage (between $2.9 \%-52 \%$ ) of Spanish oncologists are unaware of certain concepts of potential clinical significance, such as the actions of drugs upon the different opioid receptors.

The classification of breakthrough pain in Table 1 reflects potential therapeutic differences and the possible prevention of some cases through opioid use, such as for procedural breakthrough pain. As an example, pain related to cough or constipation can be improved with an antitussive agent or laxative, respectively; whereas pain related to joint movement, in some cases, can be improved by limiting mobility of the joint. ${ }^{13}$ In turn, spontaneous muscle contractions of hollow organs (esophagus, bowel, gallbladder and bile ducts, and bladder and ureters) usually cause transient and paroxysmal pain exacerbations. Colon and bladder tenesmus in turn, are the result of attempted defecation and micturition. ${ }^{32}$

The control of breakthrough pain is complicated because use is commonly made of normal-release oral opioids (such as morphine, oxycodone, and hydromorphone), the pharmacokinetic characteristics of these drugs not being optimum for treating breakthrough pain. ${ }^{11}$ The clinical and dynamic characteristics of breakthrough pain suggest that the response to an oral drug may not be optimum. ${ }^{11,33}$ The analgesic effect of an oral opioid may take 30-40 minutes, while the maximum intensity of breakthrough pain tends to be reached very quickly (sometimes in only $3-5$ minutes). ${ }^{11}$ Parenteral opioids can afford rapid analgesia, but it is not always possible to use this administration route in the home setting. ${ }^{33}$ In addition to the discrepancy between the onset of action of oral opioids and the breakthrough pain profile, these analgesics have prolonged effects (3-6 hours) and can give rise to prolonged or delayed adverse effects. ${ }^{19}$ There are five characteristics in ideal breakthrough pain drug treatment: sufficiently potent analgesia, rapid onset of action, short-lasting effect, minimum side effects, and easy administration. ${ }^{11,19}$

Drug treatment for breakthrough cancer pain (BCP) has undergone undeniable modification in recent years. The initial introduction of oral transmucosal fentanyl citrate, followed by pill and tablet formulations, and more recently, a pectinbased intranasal formulation, has considerably improved the range of treatment options. The new fentanyl formulations adapt more precisely to the profile of breakthrough pain and afford improved efficacy and a better toxicity profile (limiting overdose). A key feature of the new fentanyl formulations is their rapid onset of action, particularly considering that most breakthrough pain episodes have a duration of less than 30 minutes. In contrast, such performance is not afforded by the rapid-release formulations of classical opioids, such as morphine or oxycodone.

According to the recommendations of the Association for Palliative Medicine of Great Britain and Ireland ${ }^{34}$ and the European Association for Palliative Care (EAPC) guidelines, ${ }^{35}$ breakthrough pain should be specifically evaluated to try to establish its etiology, physiopathology, and any factor indicating or contraindicating specific interventions ${ }^{34}$ and should be effectively treated with immediate-release oral opioids or with oral or intranasal fentanyl formulations. ${ }^{35}$ The Spanish Society of Medical Oncology recently issued recommendations for the treatment of cancer pain that clearly indicate that although immediate-release morphine has been the traditional treatment for breakthrough pain, its mechanism of action does not conform to the defining characteristics of this type of pain. ${ }^{36}$ The EAPC guidelines state that oral transmucosal fentanyl formulations are more effective than immediate-release oral morphine and that intranasal fentanyl affords faster analgesia than the oral transmucosal formulation. ${ }^{35}$ It must be remembered that patients with different types of tumors who receive chemotherapy and/or radiotherapy may experience mucositis as a complication of such treatments. The pain related to mucositis prevents patients from taking oral medication or swallowing the medication and leads to a reduction in fluid and food intake. ${ }^{32}$ Other complications that also impede oral intake in cancer patients are anorexia, nausea, vomiting, dysphagia, diarrhea, constipation, stomatitis, depression, anxiety, etc.

The fentanyl doses for treating BCP episodes are not correlated to the basal analgesia. It is therefore necessary to titrate the drug dose rapidly and correctly in order to ensure patient safety and optimize treatment effectiveness. Most studies show that titration can be performed rapidly in a few BCP episodes and that in most patients, adequate titration is possible 
Table 2 Characteristics of the rapid-onset fentanyls ${ }^{36}$

\begin{tabular}{|c|c|c|c|c|}
\hline & Actiq $^{\circledR *}$ & Effentora ${ }^{\circledR *}$ & Abstral ${ }^{\circledR *}$ & Pecfent $^{\circledR *}$ \\
\hline Application & Oral transmucosal applicator & Oral transmucosal tablet & Sublingual tablet & Intranasal spray \\
\hline Time of application & $15 \mathrm{~min}$ & $14-25 \min$ & Immediate & Inmediate \\
\hline Time to onset of analgesia & $15 \mathrm{~min}$ & $10 \mathrm{~min}$ & $10 \mathrm{~min}$ & $5 \mathrm{~min}$ \\
\hline Duration of effect & No record & No record & No record & $\mathrm{I}-2 \mathrm{~h}$ \\
\hline Time until second dose & 30 min from start & $30 \mathrm{~min}$ & $15-30 \mathrm{~min}$ & $30 \mathrm{~min}$ \\
\hline Bioavailability & $50 \%$ (absolute) & $65 \%$ (absolute) & $70 \%$ (estimated) & $\begin{array}{l}120 \% \text { in respect } \\
\text { to OTFC (relative) }\end{array}$ \\
\hline Self-regulating & Yes & No & No & No \\
\hline Need-titration & Yes & Yes & Yes & Yes \\
\hline Need saliva & Yes & Yes & Yes & No \\
\hline Possible local toxicity & Yes & Yes & Yes & Yes \\
\hline
\end{tabular}

Note: *Data taken from the Summary of Product Characteristics. (2) 2012, Doyma. Reproduced with permission from Virizuela JA, Escobar Y, Cassinello J, Borrega P. Treatment of cancer pain: Spanish Society of Medical Oncology (SEOM) recommendations for clinical practice. Clin Transl Oncol. 2012;14(7):499-504.

Abbreviation: OTFC, oral transmucosal fentanyl citrate.

without having to resort to rescue analgesia before introducing fentanyl. Table 2 lists the different types of fentanyl.

The pectin-based intranasal formulation allows convenient administration of the drug, regardless of the condition of the oral mucosa of the patient. Furthermore, it provides rapid and effective analgesia (starting 5 minutes after administration, with clinically significant pain relief after 10 minutes) and can easily be administered by a caregiver if the patient is unable to collaborate, avoiding the need for training and care of the subcutaneous route if the patient is treated at home.

Despite the abovementioned specific characteristics of breakthrough pain and its ideal treatment, a study of cancer patients from four northern European countries reported that $96 \%$ of the subjects who received immediate-release morphine for the treatment of basal pain also received morphine for breakthrough pain, and likewise, $96 \%$ of the patients who received oxycodone for basal pain also received immediaterelease oxycodone for breakthrough pain. ${ }^{19}$

Some healthcare professionals, including oncologists, tend to centre priority almost exclusively on curative cancer therapy, with a lesser emphasis on palliative care - the latter being understood as the management of cancer-related symptoms such as pain. This is reflected in lesser dedication to detecting pain problems through the guided questioning of cancer patients. Many patients therefore feel hopeless and may think that physicians are not particularly concerned about their quality of life or that physicians do not understand or do not know how to treat the pain. ${ }^{3}$

\section{Conclusions and future directions}

$\mathrm{BCP}$ has an undeniable impact upon patient quality of life. Until only a few years ago consensus was lacking on the definition and classification of BCP. This has caused great discrepancy and contradiction in the data referring to the prevalence of $\mathrm{BCP}$, limited awareness of its existence as a fundamental concern in cancer patients, and suboptimum treatment of the problem. However, in recent years there has been renewed interest in this field: the definition of BCP has been clarified, its taxonomy has been established, and the basis for its treatment has been defined. ${ }^{37}$

The management of $\mathrm{BCP}$ requires an interdisciplinary approach that includes all the actors involved in the cancer patient's treatment. Since BCP affects the patient throughout the course of the disease, all specialists dealing with cancer must be familiarized with its detection and management - not only the specialist with an active part in the treatment but also in primary care. On the other hand, the management of $\mathrm{BCP}$ should be multimodal. An integrated strategy is required that includes the availability of cancer-specific treatment, appropriate analgesic use, careful control of basal pain, and the adequate indication of interventional pain procedures.

Drug treatment for BCP has undergone a genuine revolution in recent years. The new fentanyl formulations adapt more precisely to the profile of breakthrough pain and afford improved efficacy and a better toxicity profile (limiting overdose). This fact allows patients to have a better quality of life. Titration of the fentanyl treatment for BCP should be done in order to establish an adequate dose because fentanyl doses for treating $\mathrm{BCP}$ episodes are not correlated to basal analgesia (although in opioid-tolerant patients, there is no evidence for the use of dose titration in their management). Some authors have proposed establishment of doses that are proportional to basal opioid regimens for background pain because this seems to be effective and safe in the majority of patients. ${ }^{38-40}$ It is therefore necessary to titrate the drug dose rapidly and correctly in order to ensure patient safety and optimize treatment effectiveness. Nurses, caregivers and pharmacists should be involved in pain treatment education on a daily basis. 
Pain relief is related to quality of life and new treatments that allow BCP control would provide a better quality of life in cancer patients. Fentanyl-based rapid-onset treatments enable better efficacy in pain relief and less toxicity than oral treatments for BCP. We believe they should be included in all therapeutic schedules regarding cancer patients.

The great challenge in the immediate future is to efficiently deliver the knowledge gained from $\mathrm{BCP}$ to all those implicated in the cancer process and to improve our understanding with more and better studies that answer those questions for which we still do not have an answer, including: Are there clinically significant differences between the different fentanyl formulations? Can the new fentanyl formulations be as effective as the intravenous administration of opioids? Can they be administered in the absence of basal opioid treatment? Is breakthrough pain adequately diagnosed? Should medical training programs in breakthrough pain be implemented? In the near future we will undoubtedly witness further advances in all these pending areas and challenges.

\section{Acknowledgment}

The authors wish to thank Archimedes Pharma for their assistance in the preparation of this manuscript.

\section{Disclosure}

The authors state that they have no conflicts of interest.

\section{References}

1. WHO Expert Committee. Cancer Pain Relief. Geneva: World Health Organization; 1986.

2. Reyes Chiquete D, González Ortiz JC, Mohar Betancourt A, Meneses García A. Epidemiología del dolor por cancer [Epidemiology of cancer pain]. Rev Soc Esp Dolor. 2011;18(2):118-134. Spanish.

3. Breivik H, Cherny N, Collett B, et al. Cancer-related pain: a panEuropean survey of prevalence, treatment, and patient attitudes. Ann Oncol. 2009;20(8):1420-1433.

4. Yang P, Cheville AL, Wampfler JA, et al. Quality of life and symptom burden among long-term lung cancer survivors. J Thorac Oncol. 2012; $7(1): 64-70$

5. González-Escalada JR, Camba A, Casas A, et al. Código de buena práctica para el control del dolor oncológico [Good practice code for the management of cancer pain]. Rev Soc Esp Dolor. 2011;18(2):98-117. Spanish.

6. Ministerio de Sanidad y Consumo de España. Estrategia en Cáncer del Sistema Nacional de Salud. Madrid: Ministerio de Sanidad y Consumo de España; 2006. Spanish. Available from: http://www.msps.es/organizacion/sns/planCalidadSNS/pdf/ActualizacionEstrategiaCancer.pdf. Accessed September 28, 2012.

7. Palesh OG, Collie K, Batiuchok D, et al. A longitudinal study of depression, pain, and stress as predictors of sleep disturbance among women with metastatic breast cancer. Biol Psychol. 2007;75(1):37-44.

8. Scharpf J, Karnell LH, Christensen AJ, Funk GF. The role of pain in head and neck cancer recurrence and survivorship. Arch Otolaryngol Head Neck Surg. 2009;135(8):789-794.

9. Portenoy RK, Hagen NA. Breakthrough pain: definition, prevalence and characteristics. Pain. 1990;41(3):273-281.
10. Portenoy RK, Payne D, Jacobsen P. Breakthrough pain: characteristics and impact in patients with cancer pain. Pain. 1999;81(1-2):129-134.

11. Zeppetella G. Breakthrough pain should be distinguished from background pain. Guidelines in Practice. 2009;12(3):1-6.

12. Caraceni A, Martini C, Zecca E, et al; Working Group of an IASP Task Force on Cancer Pain. Breakthrough pain characteristics and syndromes in patients with cancer pain. An international survey. Palliat Med. 2004;18(3):177-183.

13. Zeppetella G. Impact and management of breakthrough pain in cancer. Curr Opin Support Palliat Care. 2009;3(1):1-6.

14. Davies AN. The management of breakthrough cancer pain. Br J Nurs. 2011:20(13):803-807.

15. Dickman A. Integrated strategies for the successful management of breakthrough cancer pain. Curr Opin Support Palliat Care. 2011; 5(1):8-14.

16. Davies AN, Dickman A, Reid C, Stevens AM, Zeppetella G; Science Committee of the Association for Palliative Medicine of Great Britain and Ireland. The management of cancer-related breakthrough pain: recommendations of a task group of the Science Committee of the Association for Palliative Medicine of Great Britain and Ireland. Eur J Pain. 2009;13(4):331-338.

17. Porta-Sales J, Garzón Rodríguez C, Julià Torras J, Casals Merchán M. Dolor irruptivo en cáncer [Cancer-related breakthrough pain]. Med Clin (Barc). 2010;135(6):280-285. Spanish.

18. Swanwick M, Haworth M, Lennard RF. The prevalence of episodic pain in cancer: a survey of hospice patients on admission. Palliat Med. 2001;15(1):9-18.

19. Davies A, Zeppetella G, Andersen S, et al. Multi-centre European study of breakthrough cancer pain: pain characteristics and patient perceptions of current and potential management strategies. Eur J Pain. 2011;15(7): 756-763.

20. American Pain Foundation. Breakthrough cancer pain: mending the break in the continuum of care. J Pain Palliat Care Pharmacother. 2011;25(3):252-264.

21. Svendsen KB, Andersen S, Arnason S, et al. Breakthrough pain in malignant and non-malignant diseases: a review of prevalence, characteristics and mechanisms. Eur J Pain. 2005;9(2):195-206.

22. Howell J, Dumble S. Quality of life in patients with breaththrough cancer pain: results from the two phase III studies of sublingual fentanyl. European Journal of Pain Supplements. 2011;5:171. doi 10.1016/ S1754-3207(11)70588-0.

23. Breuer B, Fleishman SB, Cruciani RA, Portenoy RK. Medical oncologists' attitudes and practice in cancer pain management: a national survey. J Clin Oncol. 2011;29(36):4769-4775.

24. van den Beuken-van Everdingen MH, de Rijke JM, Kessels AG, Schouten HC, van Kleef M, Patijn J. Prevalence of pain in patients with cancer: a systematic review of the past 40 years. Ann Oncol. 2007;18(9): 1437-1449.

25. Bernabei R, Gambassi G, Lapane K, et al. Management of pain in elderly patients with cancer. SAGE Study Group. Systematic Assessment of Geriatric Drug Use via Epidemiology. JAMA. 1998;279(23):1877-1882.

26. Gómez-Batiste X, Madrid F, Moreno F, et al. Breakthrough cancer pain: prevalence and characteristics in patients in Catalonia, Spain. J Pain Symptom Manage. 2002;24(1):45-52.

27. Fortner BV, Okon TA, Portenoy RK. A survey of pain-related hospitalizations, emergency department visits, and physician office visits reported by cancer patients with and without history of breakthrough pain. J Pain. 2002;3(1):38-44.

28. American Pain Foundation. Breakthrough Cancer Pain Survey Fact Sheet. American Pain Foundation; 2012. Available from: http://nci nih.gov/dictionary/?CdrID=45612. Accessed June 12, 2012.

29. Von Roenn JH, Cleeland CS, Gonin R, Hatfield AK, Pandya KJ. Physician attitudes and practice in cancer pain management. A survey from the Eastern Cooperative Oncology Group. Ann Intern Med. 1993;119(2):121-126.

30. Carulla Torrent J, Jara Sánchez C, Sanz Ortiz J, el al; ALGOS Group. Oncologists' perceptions of cancer pain management in Spain: the real and the ideal. Eur J Pain. 2007;11(3):352-359. 
31. Escobar Álvarez Y, Rodríguez Sánchez C, Caballero Martínez F, Recuero Cuervo V, Camps Herrero C. Professional survey on knowledge and clinical patterns of pain management in Spanish medical oncology. Clin Transl Oncol. 2010;12(12):819-824.

32. Mercadante S, Radbruch L, Caraceni A, et al. Episodic (breakthrough) pain: consensus conference of an expert working group of the European Association for Palliative Care. Cancer. 2002;94(3):832-839.

33. Mercadante $\mathrm{S}$. The use of rapid onset opioids for breakthrough cancer pain: the challenge of its dosing. Crit Rev Oncol Hematol. 2011;80(3): $460-465$.

34. breakthroughcancerpain.org. APM recomendations. Association for Palliative Medicine of Great Britain and Ireland; 2009. Available from: http://www.breakthroughcancerpain.org/pain-management/ apm-recommendations Accessed June 12, 2012.

35. Caraceni A, Hanks G, Kaasa S, et al; European Palliative Care Research Collaborative (EPCRC); European Association for Palliative Care (EAPC). Use of opioid analgesics in the treatment of cancer pain: evidence-based recommendations from the EAPC. Lancet Oncol. 2012; 13(2):e58-e68.
36. Virizuela JA, Escobar Y, Cassinello J, Borrega P. Treatment of cancer pain: Spanish Society of Medical Oncology (SEOM) recommendations for clinical practice. Clin Transl Oncol. 2012;14(7):499-504.

37. LeBlanc TW, Back AL. Linking palliative care and oncology practice: performance status as a common thread. J Oncol Pract. 2011;7(6): 381-382.

38. Mercadante S, Gatti A, Porzio G, et al. Dosing fentanyl buccal tablet for breakthrough cancer pain: dose titration versus proportional doses. Curr Med Res Opin. 2012;28(6):963-968.

39. Mercadante S. Oral trasmucosal fentanyl citrate for breakthrough pain in treatment in cancer patients. Expert Opin Pharmacother. 2012;13(6):873-878.

40. Mercadante S. Pharmacotherapy for breakthrough cancer pain. Drugs. 2012;72(2):181-190
Journal of Pain Research

\section{Publish your work in this journal}

The Journal of Pain Research is an international, peer-reviewed, open access, online journal that welcomes laboratory and clinical findings in the fields of pain research and the prevention and management of pain. Original research, reviews, symposium reports, hypothesis formation and commentaries are all considered for publication.

\section{Dovepress}

The manuscript management system is completely online and includes a very quick and fair peer-review system, which is all easy to use. Visit http://www.dovepress.com/testimonials.php to read real quotes from published authors. 